\title{
Dgcr8 is Indispensable for Cardiac Lineage Specification in Embryonic Stem Cells
}

\author{
Zhiyong Lei ${ }^{1}$, Alain van Mili ${ }^{1}$, Annebel M van de Vrugt ${ }^{1}$, Pieter A Doevendans ${ }^{1,2}$ and Joost PG Sluijter ${ }^{1,2 *}$ \\ ${ }^{1}$ Department of Cardiology, Division Heart and Lungs, University Medical Center Utrecht, Utrecht, the Netherlands \\ ${ }^{2}$ ICIN, Netherlands Heart Institute, Moree/separk 1, 3511 EP, Utrecht, the Netherlands
}

\begin{abstract}
Objective: microRNAs have been shown to play important roles in cellular behavior and lineage specification including cardiogenic differentiation. However, full understanding of their roles in cardiomyocyte differentiation has been impeded due to lack of proper cellular models. Here, we used an embryonic stem cell (ESC) that is lacking the important microprocessor Dgcr8 (or Pasha), which allows the introduction of individual miRNAs to study their role in cardiac differentiation and for more precise target selection.
\end{abstract}

Methods: Dgcr8 KO ESC was cultured in LIF-supplemented ESC medium with mouse embryonic fibroblast feeders and cardiac differentiation was induced using an embryonic body-based differentiation protocol. Differentiation was monitored by measuring mRNA and protein levels of cardiogenic markers and heterochromatin changes using immunofluorescent staining and semi-quantitative PCR.

Results and conclusion: We showed that Dgcr8 KO ESCs indeed are lacking a large population of small RNAs, including but not limited to mature microRNAs. The KO cells had a lower proliferation rate and were unable to differentiate into the cardiac lineage. To our surprise, in addition to a defect in microRNA processing, Dgcr8 KO embryonic stem cells are unable to form proper heterochromatin and to inactivate genotoxic centromeric repetitive elements. Our results argue that, in addition to controlling microRNA processing, Dgcr8 may serve a previously unrecognized role in heterochromatin silencing.

Keywords: Embryonic stem cell differentiation; Dgcr8; Heterochromatin silencing

Abbreviations: Dgcr8: DiGeorge syndrome critical region 8; ESC: Embryonic Stem Cell; ncRNA: noncoding RNA; RITS: RNAi Induced Transcriptional Silencing Complex; MEF: Mouse Embryonic Fibroblast; EB: Embryonic Body; RT-PCR: Reverse Transcription Polymerase Chain Reaction; WT: wildtype; KO: knockout; RBL2: Retinoblastomalike 2 protein; RISC: RNAi Induced Silencing Complex; PTGS: Post Transcriptional Gene Silcencing; CDGS: Chromatin Dependent Gene Silencing; FOA: Fetal Oocyte Attrition; TnT: Troponin T

\section{Introduction}

MicroRNAs (miRNAs) have been shown to play important roles in cardiac differentiation [1-6], pathological development and progression of cardiac diseases [7-12]. Full understanding of their role in these conditions requires identification of their precise target genes and studying their function in the relevant cell types. Several different cell types, such as HEK293 or COS7, or organs like the mouse heart have been used for identification and validation of different miRNA targets $[13,14]$. A major hurdle for target identification is that the cells used are either not relevant for the studied process, thereby not having the correct target mRNAs present, or the redundancy caused by other miRNAs that may compete with the miRNA of interest and thereby interfere with the effect of overexpression or inhibition of the miRNA of interest $[15,16]$. Therefore, an ideal cell model to study the effects of a single miRNA including its targets in a cardiac context would be a cardiomyocyte without any mature miRNAs present, a very "Simple Cell" as it were.

Most of the currently known miRNAs go through canonical miRNA processing. They are transcribed as normal mRNAs by RNA Polymerase II and subsequently processed into mature miRNAs by microprocessor complexes including Dgcr8 and Drosha [17]. After being exported into the cytosol $[18,19]$, precursor miRNAs are further processed into mature miRNAs by Dicer [20,21]. Therefore, cells lacking Dgcr8 or Dicer are not able to produce canonical mature miRNAs. Dicer has been shown to have miRNA independent roles and commercial miRNA mimics are provided as precursor or precursor like structures, which require processing by Dicer to become functional. Thus, knocking out Dgcr8 in embryonic stem cells (ESC) has become the first choice to create a versatile miRNA-free cell model. In this cell model, the miRNA population will be eliminated, however, the activity for precursor processing will be kept intact and the cells may hold their potential to differentiate into any cell type of interest, e.g. cardiomyocytes. Although inactivation of Dgcr8 was used to explore the role of the total miRNA population in a particular cell type or tissue in vivo [22-25], the consequence of the loss of Dgcr8 on cardiac lineage specification of ESCs, and whether or not we can create the "simple cardiomyocyte" from Dgcr8 KO ESCs, is unknown.

\section{Methods and Materials}

Embryonic stem cell (ESC) culture and embryonic bodies (EB) preparation and differentiation

Wild-type ESCs V6.5 and Dgcr8 knockout (KO) ESCs (Novus

*Corresponding author: Joost PG Sluijter, Division of Heart and Lungs, Department of Cardiology, Experimental Cardiology Laboratory, Huispostnummer G02.523, Postbus 85500, 3508 GA Utrecht, the Netherlands, Tel: + 3188755 7567; Fax: + 3130252 2693; E-mail: j.sluijter@umcutrecht.nl

Received December 15, 2014; Accepted January 13, 2015; Published January 15,2015

Citation: Lei Z, van Mil A, van de Vrugt AM, Doevendans PA, Sluijter JPG (2015) Dgcr8 is Indispensable for Cardiac Lineage Specification in Embryonic Stem Cells. J Stem Cell Res Ther 5: 260. doi:10.4172/2157-7633.1000260

Copyright: (c) 2015 Lei Z, et al. This is an open-access article distributed under the terms of the Creative Commons Attribution License, which permits unrestricted use, distribution, and reproduction in any medium, provided the original author and source are credited. 
Citation: Lei Z, van Mil A, van de Vrugt AM, Doevendans PA, Sluijter JPG (2015) Dgcr8 is Indispensable for Cardiac Lineage Specification in Embryonic Stem Cells. J Stem Cell Res Ther 5: 260. doi:10.4172/2157-7633.1000260

Page 2 of 7

Biologicals, NBA1-19349) were cultured on a MEF feeding layer in ESC medium, containing DMEM (Gibco, cat. no. 41965), 15\% FBS (Gibco, cat. no. 16141-079), 1x penicillin/streptomycin (Sigma, cat. no. P4458), 1x MEM-nonessential amino acids (PAA, cat. no. M11003), $1 \mathrm{x}$ Leukemia Inhibitory Factor, and $0.0007 \% \beta$-mercaptoethanol. Cells were detached using Accutase (Chemicon Millipore, SF006) and before differentiation, preplated for $0.5-2 \mathrm{~h}$ in non-coated plates. The supernatant including ESCs was collected, and EBs were formed in low attachment plates (6 well) with 2 million cells per well in differentiation medium, containing IMDM (Gibco, cat. no. 21980), Ham's F12 nutrient mixture with GlutaMAX-I (Gibco, cat. no. 31765), 2\% Horse serum (Gibco, 16050-122), 1x MEM-nonessential amino acids (BioWhittaker, BE13-114E), 1x Insulin transferrin-selenium (Gibco, 41400-045), 1x penicillin/streptomycin (Sigma, cat. no. P4458), and $25 \mu \mathrm{g} / \mathrm{mL}$ ascorbic acid (Sigma, A-4034). The cells were incubated at $37^{\circ} \mathrm{C}$ while shaking (circa 50 rotations/min) for 2 days to induce EB formation.

\section{Proliferation rate assay}

To determine the degree of proliferation, cells were trypsinized into single cells and 10,000 cells were seeded in a 24 -well plate, pre-coated with MEFs. Cells were trypsinized into single cells at 48 and 72 hours after seeding and counted with a Bio-Rad TC-10 automatic cell counter.

\section{RNA isolation, small RNA profiling and semi-quantitative PCR analysis}

Total DNA-free RNA was isolated with Tripure Isolation Reagent following manufactory's instructions (Roche Applied Science) and analyzed by using the Agilent Small RNA Kit (Agilent Technologies, cat. no. 5067-1548) according to the manufacturer's guidelines. Results were obtained using the Agilent Bioanalyzer 2100 and data was analyzed with 2100 Expert Software.

Total RNA was treated with RNase-free DNase I (Qiagen), checked to be DNA free, and cDNA was made using the iScript ${ }^{\mathrm{TM}} \mathrm{cDNA}$ Synthesis Kit (Bio-Rad). Quantitative real-time polymerase chain reaction (qRT-PCR) was performed with iQ SYBR Green Supermix (Bio-Rad) and specific primers with the following sequences: Gapdh forward 5'-GGCATGGACTGTGGTCATGA-3' and reverse 5'-TTCACCACCATGGAGAAGGC-3'; Hprt forward 5' TCAGTCAACGGGGGACATAAA-3' and reverse 5'- GGGGCTGTACTGCTTAACCAG -3'; Oct4 forward 5'- AGAGGATCACCTTGGGGTACA-3' and reverse 5'- CGAAGCGACAGATGGTGGTC -3'; Sox2 forward 5'- GCGGAGTGGAAACTTTTGTCC-3' and reverse 5'- CGGGAAGCGTGTACTTATCCTT -3'; Nanog forward 5'- TCTTCCTGGTCCCCACAGTTT-3' and reverse 5'- GCAAGAATAGTTCTCGGGATGAA -3'; Hnf4 $\alpha$ forward 5'- GTGGCGAGTCCTTATGACACG-3' and reverse 5'- GCTGTTGGATGAATTGAGGTTGG -3'; TnT forward 5'- CAGAGGAGGCCAACGTAGAAG-3' and reverse 5'- CTCCATCGGGGATCTTGGGT $-3^{\prime} ; \alpha$-MHC forward 5'-ATCCTGTCCAACAAGAAGCC-3' and reverse 5'-ACCTCTCCCTGAGAGACGAA-3'; $\beta$-actin forward 5'-CTCTTTTCCAGCCTTCCTTC-3' and reverse 5'-TCTCCTTCTGCATCCTGTC-3'; Pasha exon 3 forward 5'-CACGGCTAAAGCAATCGTTCAA-3' and reverse 5'-GCTCTGTAGGTGGACGGCCAC-3'; LINE-1 forward 5'- GAACAGACGAGGACACTGAAAA-3' and reverse 5'- CTGGCTTCTCATTCGCATTCT-3'; IAP forward 5'- AGCAAGAAAAGAAGCCCGTGA-3' and reverse 5'- ATGCCAGAACATGTGTCAAGTG-3'; Major forward 5'- GACGACTTGAAAAATGACGAAATC-3' and reverse 5'- CATATTCCAGGTCCTTCAGTGTGC-3'; Minor forward 5'- CATGGAAAATGATAAAAACC-3' and reverse 5'- CATCTAATATGTTCTACAGTGTGG-3'. For minor and major satellite RNA RT-PCR, PCR products were sepa- rated using agarose gels (2\%) and images were taken using UV exposure and detection with the ChemiDoc XRS System (Bio-Rad).

\section{Western blotting}

Total protein was isolated with protein lysis buffer (Roche Applied Science). Protein amounts were determined using the Pierce BCA Protein Assay (Thermo Scientific). Equal amounts of protein were loaded, separated, and transferred to a PVDF membrane using the Novex ${ }^{\oplus}$ NuPAGE ${ }^{\oplus}$ SDS-PAGE Gel System and the iBlot ${ }^{\circledR}$ Transfer System (Invitrogen). PVDF membranes were blocked in 5\% non-fat dry milk in $0,1 \%$ TBST with $2 \%$ normal goat serum or $5 \%$ non-fat dry milk in $0,1 \%$ PBST with $2 \%$ normal goat serum. Blocked membranes were incubated with primary antibodies against Pasha (Dgcr8; 1:1000, Proteintech, cat. no. 10996-1-AP) or $\beta$-actin (1:5000, Sigma, cat. no. A5441), and subsequently probed with horseradish peroxidase-conjugated goatanti-rabbit (1:2000, Dako, P0448) or goat-anti-mouse (1:1000, Dako, P0447). The signal was visualized with Chemiluminescent Peroxidase Substrate (Sigma) and detected with the ChemiDoc XRS System (BioRad). Image Lab software was used for analysis.

\section{Fluorescent immunohistochemistry}

EBs were fixed with 4\% PFA in PBS, incubated in 30\% sucrose PBS, and preserved in Tissue-Tek ${ }^{\oplus}$ O.C.T. ${ }^{\mathrm{TM}}$ (Sakura). Cryosections were stained for $\alpha$-actinin (1:800, Sigma, cat. no. A7811), and Troponin I (1:100, Abcam, cat. no. ab47003). The secondary antibodies used were Alexa Fluor-488 goat-anti-mouse (1:400, Invitrogen, A11001) and Alexa Fluor-555 goat-anti-rabbit (1:400, Invitrogen, A21458). Nuclei were counterstained with $1 \mu \mathrm{g} / \mathrm{mL}$ Hoechst 33342 (Invitrogen) and sections were mounted in Fluoromount-G (SouthernBiotech). Images were captured using confocal laser scanning microscopy (Carl Zeiss LSM 700) and associated software.

\section{Statistical analysis}

Data are presented as mean \pm SEM. Student's t-tests were performed for comparison between WT and $\mathrm{KO}$ and differences were considered statistically significant when $\mathrm{p}$ values were $<0.05$, as indicated by an asterisk $\left({ }^{*}\right)$.

\section{Results}

\section{Characterization of mouse Dgcr8 KO ESCs}

To confirm the absence of Dgcr8 in KO ESCs, we designed primers which amplify the 3 ' part of intron 2 and the $5^{\prime}$ part of intron 4 and performed a PCR with WT and KO genomic DNA. As expected, PCR products from $\mathrm{KO}$ are smaller (100 bp) compared with WT products (248 bp) (Figures 1A and B). Additionally, we performed Western blotting with an anti-Dgcr8 antibody with protein lysate of WT and KO ESC. We indeed confirmed the absence of the predicted $86 \mathrm{kD}$-signal in the KO samples (Figure 1C). The loss of Dgcr8 in the KO ESC was further confirmed by immunofluorescent staining, as is illustrated by the absence of nuclear Dgcr8 in KO ES cells (Figure 1D).

Dgcr8 KO ESCs were viable and displayed typical mouse ESC morphology when cultured on a mouse embryonic fibroblast feeder layer. Interestingly, KO ESC colonies were smaller as compared with WT colonies (Figure 1E). And we also noticed Dgcr8 KO ESCs proliferate much slower than WT cells (Figure 1F).

Dgcr8 was first identified as a Drosha interacting protein by Drosha immunoprecipitation, and therefore its function was mainly associated with miRNA biogenesis. To evaluate the consequence of Dgcr8 loss on 
Citation: Lei Z, van Mil A, van de Vrugt AM, Doevendans PA, Sluijter JPG (2015) Dgcr8 is Indispensable for Cardiac Lineage Specification in Embryonic Stem Cells. J Stem Cell Res Ther 5: 260. doi:10.4172/2157-7633.1000260

A

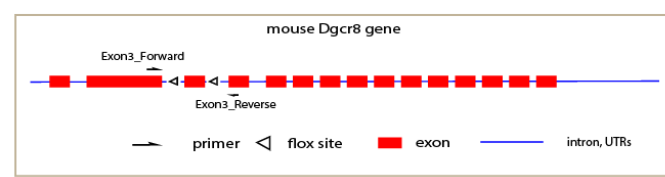

B
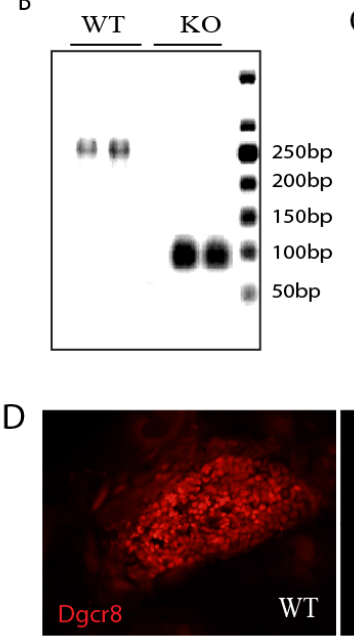

C

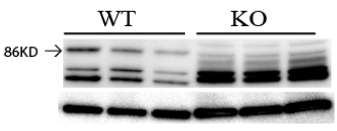

WT

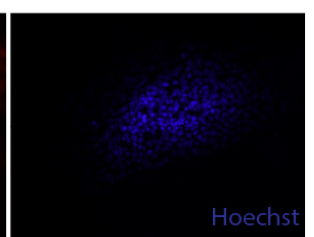

KO

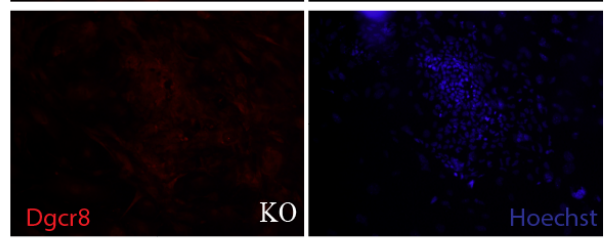

$\mathrm{E}$
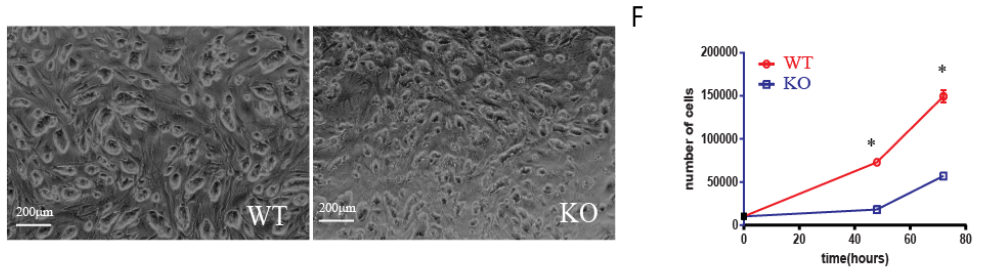

G

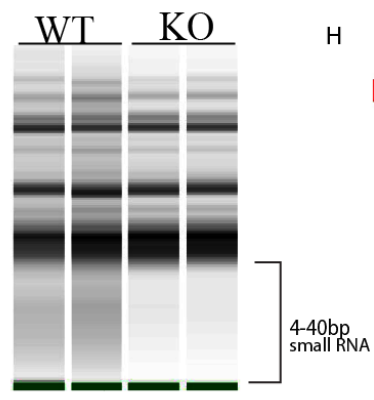

H

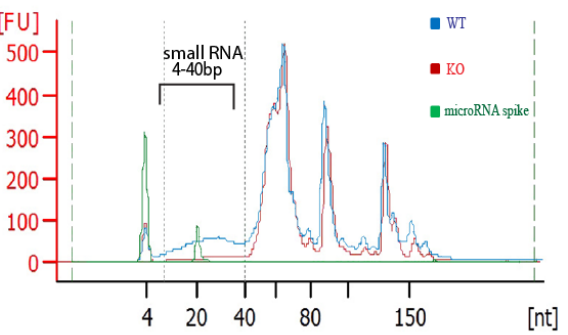

Figure 1: Characterization of Dgcr8 KO mouse embryonic stem cell,

A. Illustration of the mouse Dgcr8 gene, including the locations of 2 flox sites that were used for gene targeting and the design of genotyping primers, as used in figure $1 \mathrm{C}$.

B. Validation of the loss of Dgcr8 protein in the Dgcr8 KO-ESCs by Western blotting.

C. Genotyping of WT and KO ESCs with primers franking exon2 and exon4. WT display a band at $248 \mathrm{bp}$ and KO at $100 \mathrm{bp}$

D. Immunofluorescent staining of Dgcr8 on WT and KO ESC colonies. Note the nuclear staining of dgcr8 in the WT colony.

E. Representative images of WT and Dgcr8 KO ESCs in culture.

F. Comparison of WT and Dgcr8 KO ESC proliferation rate in culture.

G. Representative images of total RNA profiles from WT and Dgcr8 KO ESCs using bioanalyzer 2100. Note the absence of small RNA species in Dgcr8 KO ESCs, ranging from 4-40 nt long

$\mathrm{H}$. Histogram of the profiles of the total RNA from WT and Dgcr8 KO ESCs using bioanalyzer 2100. Note the absent of small RNA species in Dgcr8 KO ESCs ranging from 4-40 nt long.

the cellular RNA profile, we isolated total RNA from WT and KO ESCs and compare the small RNA profiles (1-150 bp) using a Bioanalyzer small RNA chip. We observed that Dgcr8 KO cells are not only lacking miRNAs, as indicated by the miRNA spike in control (20 bp), but also a large population of small RNA species ranging from 4-40 bp (Figure $1 \mathrm{G}$ and $1 \mathrm{H})$.

\section{Dgcr8 KO EBs do not differentiate into the cardiac lineage}

To assess the effect of the Dgcr8 KO on the cardiogenic differentiation of ESCs, we induced cardiac differentiation using a wellestablished EB-based differentiation protocol [26]. We observed that Dgcr8 KO ESCs can form EBs, but their size is smaller and their shape is more irregular compared to WT ESC derived EBs (Figure 2A). Upon induction of differentiation, WT ESCs undergo typical morphological and phenotypical changes, such as forming of cyst-like structures and spontaneous contraction (Figure $2 \mathrm{~B}$ ). In contrast, $\mathrm{KO}$ EBs did not show cyst-like structures and did not contract (Figure 2A and 2B).
To further characterize the cardiogenic differentiation of EBs, we performed qRT-PCR for pluripotency (Oct4), early mesoderm (Brachyury $\mathrm{T}$ ), and cardiogenic lineage markers (aMHC and $\mathrm{TnT}$ ). Consistent with our previous observations, we found that the expression of Oct4 decreased upon differentiation, associated with an increased level of Brachyury T in WT cells. Prolonging the differentiation reduced the expression of Brachyury $\mathrm{T}$ and increased the levels of cardiac markers aMHC and Troponin in WT EBs on day 10. These results indicate loss of pluripotency and induced mesoderm and subsequent cardiac differentiation in the WT EBs. In the Dgcr8 KO cells, however, the expression of pluripotency markers Oct4, Sox2, and Nanog did not change, only very low levels of Brachyury T could be detected, and the expression of aMHC and Troponin was absent, even 10 days after differentiation (Figure 2C). We further confirmed these findings by immunofluorescent staining of EBs for the cardiac markers $\alpha$-Actinin and Troponin. Indeed, we could detect $\alpha$-Actinin and Troponin positive regions in the WT EBs 
Citation: Lei Z, van Mil A, van de Vrugt AM, Doevendans PA, Sluijter JPG (2015) Dgcr8 is Indispensable for Cardiac Lineage Specification in Embryonic Stem Cells. J Stem Cell Res Ther 5: 260. doi:10.4172/2157-7633.1000260

A

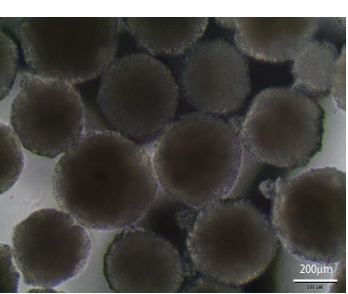

WT

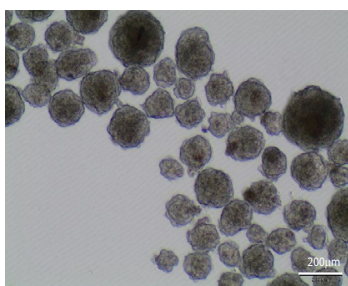

$\mathrm{KO}$ day 3

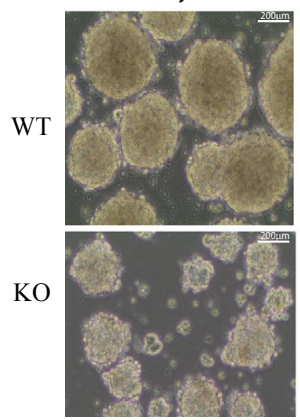

day 7

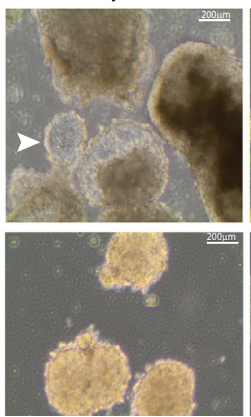

day 10

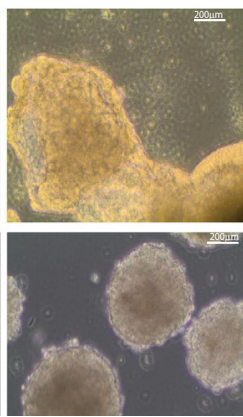

$\mathrm{C}$
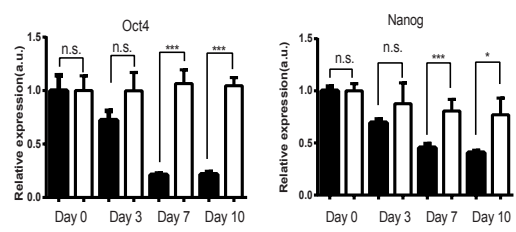

Day 0 Day 3 Day 7 Day 10
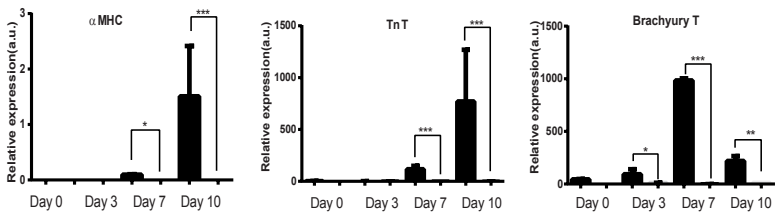
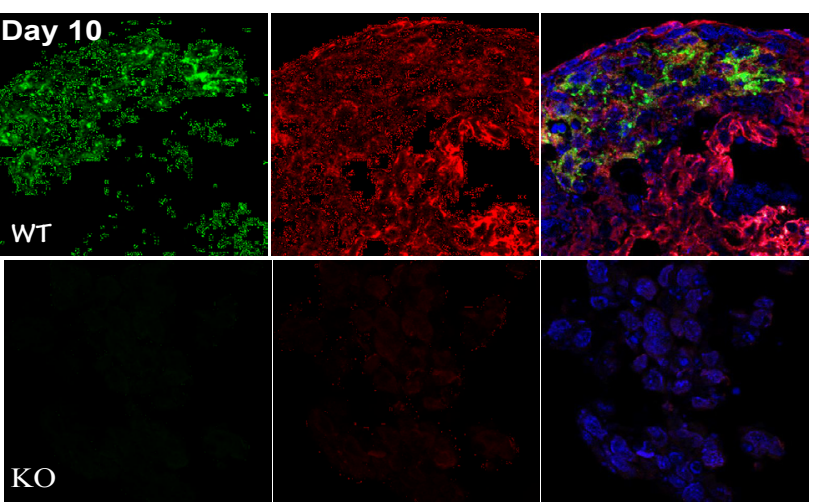

actinin/TroponinT/Hoechst

Figure 2: Dgcr8 KO ESCs are defect in cardiogenic differentiation

A. Morphology of WT and Dgcr8 KO EBs, note the irregular shape/size of Dgcr8 KO EBs.

B. Morphology of WT and Dgcr8 KO EBs 3, 7 and 10 days after initiation of differentiation. Note Dgcr8 KO EBs are smaller, and lack of cyst-like structures which are present in WT EBs after 7 and 10 days differentiation.

C. qPCR analysis of Oct4, a stem cell pluripotency marker, and differentiation marker Brachyury T, Troponin T(TnT) and $\alpha M H C$

D. Immunofluorescent staining for cardiac marker $\alpha$-Actinin and Troponin T in WT and KO cells after 10 days of differentiation.

after 10 days of differentiation, which were not observed in the KO EBs (Figure 2D).

\section{Dgcr8-KO ESCs are deficient in heterochromatin silencing and centromeric repeat sequence RNA inactivation}

Although miRNA processing was the first role ascribed to Dicer, defects in the formation of heterochromatin have been linked to failure of differentiation and induction of apoptosis in Dicer KO ESCs [27,28]. Centromeric heterochromatin regions contain a lot of transposable elements, which have to be silenced by the formation of heterochromatin rich regions. RNAi mediated heterochromatin assembly has been reported to play a critical role in ESC differentiation, genome stability and protection from viral invasion [29]. Recently, a Dgcr8 RNA immune-precipitation study has greatly expanded its target spectrum and has shown its physical interaction with several other non-coding RNAs (ncRNA), including retro-transposable elements as well as Major Satellite and Minor Satellite centromeric repeat RNA species [30], thereby regulating retro-transposon activities [31]. Dgcr8 null ESCs are not able to produce a large population of endo-siRNA, or shRNA [32], essential components of siRNA induced chromatin silencing complexes. Therefore, we investigated if there were any differences in heterochromatin formation between WT and Dgcr8 KO ESCs. Using a H3K9ME3 antibody, a well-established heterochromatin marker [27,33], we performed immunofluorescent staining on WT and Dgcr8 KO ESCs before and after differentiation. Although we found no differences before differentiation (Figure $3 \mathrm{~A}$ ), upon differentiation we did observe a clear condensation pattern only in WT cells (Figure 3B). A clear example of heterochromatin condensation is seen in differentiated fibroblast (Figure $3 \mathrm{C}$ ). This pattern is not seen in the $\mathrm{KO}$ ESCs, suggesting there is a defect in the formation of heterochromatin regions in KO ESCs.

To further confirm this finding, we selected four centromeric repeat sequences: minor and major satellite repeats, and LINE-1, all identified as Dgcr8-interacting RNAs [30], and IAP, and performed a semiquantitative PCR using WT and KO ESC RNA samples. As predicted, we found that the expression of LINE-1 and minor and major satellite transcripts is more abundant in the KO ESCs compared to WT ESC, before and after differentiation (Figure $4 \mathrm{~A}$ and $4 \mathrm{~B}$ ). The expression of these three RNA transcripts is less pronounced upon differentiation in the WT ESCs (Figure 4B and Supplement Figure A-E), which correlates with the widespread heterochromatin formation as shown by the H3K9ME3 staining (Figure 3A and 3B). The higher expression of these three transcripts in the KO ESCs indicates a defect in heterochromatin silencing (Figure 4B and Supplement Figure E). Interestingly, another retro-transposon-like element, IAP, did not show any difference (Supplement Figure F).

\section{Discussion}

In this study, we aimed to characterize and generate a miRNAfree cell that potentially can be used for miRNA functional analysis in cardiomyocytes. Our results show that Dgcr8 KO ESCs proliferate 
Citation: Lei Z, van Mil A, van de Vrugt AM, Doevendans PA, Sluijter JPG (2015) Dgcr8 is Indispensable for Cardiac Lineage Specification in Embryonic Stem Cells. J Stem Cell Res Ther 5: 260. doi:10.4172/2157-7633.1000260
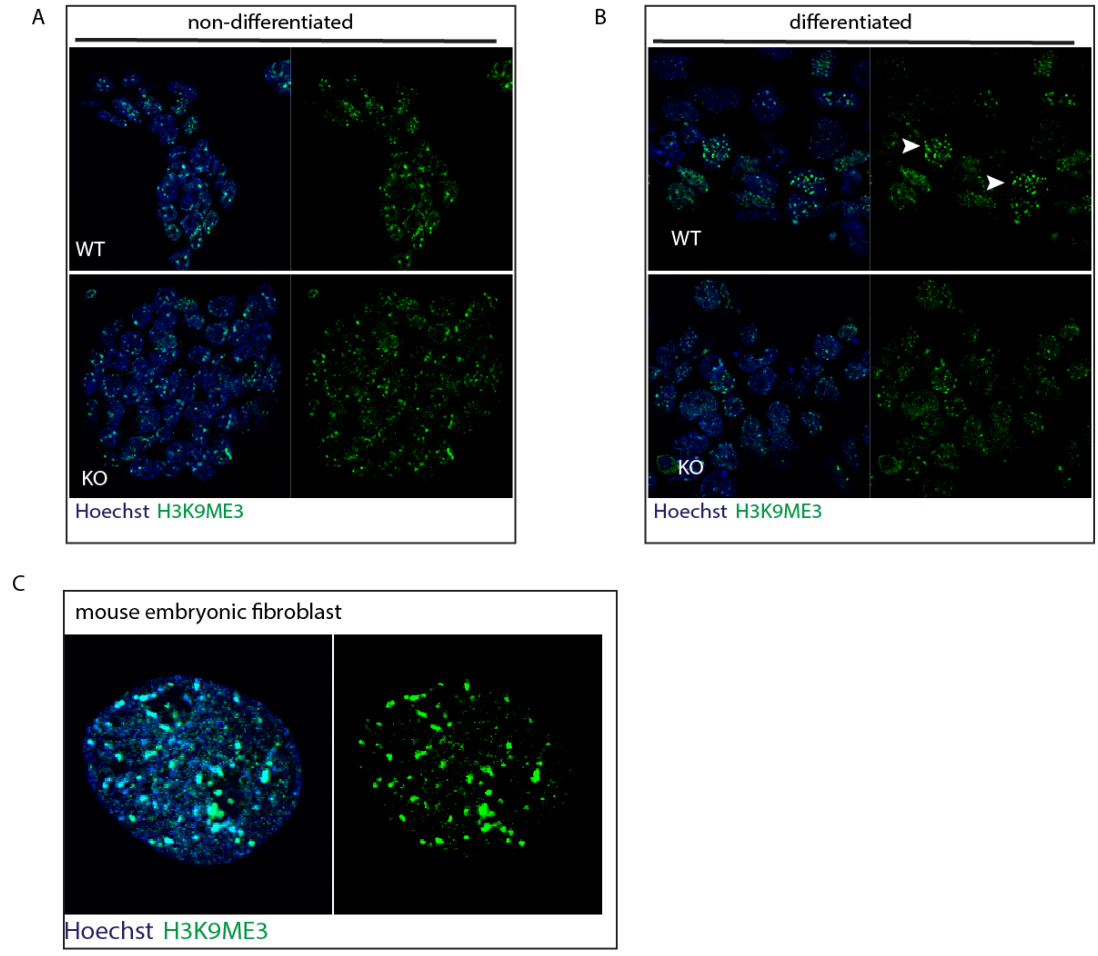

Figure 3: Dgcr8 KO ESC is deficient in heterochromatin silencing during differentiation

A. H3K9ME3 immunofluorescent staining on non-differentiated WT and KO ESC.

B. H3K9ME3 immunofluorescent staining on 7 days differentiated WT and KO ESCs. Note the H3K9ME3 condense staining in the different WT cells, as indicated with white arrows which is absent in the Dgcr8 KO ESCs.

C. H3K9ME3 immunofluorescent staining on mouse embryonic fibroblast served as a positive control.

slower and are lacking a large population of small RNA species ranging from 4-40 bp, including mature miRNAs ( $20 \mathrm{bp})$. However, Dgcr8 KO ESCs are unable to undergo cardiogenic differentiation. Furthermore, we observed that centromeric regions of KO ESCs have a higher transcriptional activity upon induction of differentiation as compared to WT controls. Therefore, Dgcr8 KO ESCs are unable to fully inactivate their heterochromatin regions like WT ESCs, which is likely leading to the defect in cardiac differentiation.

Consistent with a previous study, we found that Dgcr8 KO ESCs exhibit slower proliferation rates. It has been shown that the miR290 family is involved in the regulation of ESC proliferation [34] and overexpression of this family could partially rescue the proliferation defect of Dgcr8 KO ESCs [34]. Additionally, we observed a defect in cardiomyogenic differentiation in the Dgcr8 KO ESCs, as shown by the lack of spontaneous contraction and absence of myogenic differentiation markers which agree with previous observation that Dgcr8 KO ESC is not capable to form teratoma [34]. Dicer is also involved in miRNA processing, which is downstream of Dgcr8. Similar to Dgcr8 KO ESCs, Dicer KO ESCs display a similar phenotype [27], but show a more pronounced defect in heterochromatin silencing even before differentiation [27].

How and if microprocessors like Dgcr8 or Dicer control ESC differentiation through miRNA processing is not known. Although a lack of miRNA processing seems the most obvious explanation for the defect in differentiation, more and more studies point to a miRNA biogenesis-independent role of the microprocessor. In fission yeast, which does not have miRNAs, RNAi-mediated heterochromatin silencing has been shown to play important roles in protection of virus invasion and genome integrity. There, RNA transcripts from centromeric chromatin regions are used as a template by the RNA dependent RNA polymerase (RdRP) to generate dsRNAs which are subsequently processed into short dsRNAs. The short dsRNAs serve as a guide for the RNA-induced Silencing Complex (RISC) containing Ago-complexes for both Post-Transcriptional Gene Silencing (PTGS) and Chromatin Dependent Gene Silencing (CDGS) [29]. In mammals, small RNAs, that play an important role in silencing genotoxic transposable genetic regions [35,36], have also been identified, both in ESCs and germ cells [32]. In germ cells, for example, a class of small RNAs called Piwi-associated RNAs (piRNAs), which are longer than miRNAs (26-31 nt), have been shown to play an important role in silencing genotoxic transposable genetic regions [35,36]. These small RNAs have also been found in ESCs, however, the function of these small RNAs in ESCs is unclear. Before differentiation, centromeric chromatin regions are slightly more open in the Dgcr8 KO cells as shown by minor and major satellite RNA semi-quantitative RT-PCR. Upon differentiation, centromeric chromatin regions are inactivated in WT but not in the Dgcr8 KO cells. The functional importance of major satellite RNA transcripts is in line with a recent study, in which manipulation of major satellite RNA completely blocked embryonic differentiation at 2-cell stages[37]. Our results suggest that Dgcr8 might carry a previously unrecognized small RNA related function which is independent of miRNA biogenesis, as was also reported for Dicer [27]. Future studies are needed to fully understand its function during this process and to identify its substrates and working partners [32].

How the defective heterochromatin silencing in Dgcr8 KO ESCs prevents their differentiation into the cardiac lineage is not clear. 
Citation: Lei Z, van Mil A, van de Vrugt AM, Doevendans PA, Sluijter JPG (2015) Dgcr8 is Indispensable for Cardiac Lineage Specification in Embryonic Stem Cells. J Stem Cell Res Ther 5: 260. doi:10.4172/2157-7633.1000260

However, recent studies about LINE1 may shed some light on this question. LINE1, one of redundant retro-transposon derived RNA species, which are distributed in the heterochromatin regions across the genome, is inactivated upon differentiation. In oocytes, the level of the LINE1 is used to determine which cell will go into apoptosis, a well conserved phenomena called fetal oocyte attrition (FOA) [38]. Oocytes with low LINE1 activity are selected and the ones with high LINE1 levels are eliminated [38]. Interestingly, Dgcr8 KO ESCs show accumulation of LINE-1 and a defect in inactivation of LINE-1 upon cardiac differentiation. It is conceivable that a failure in inactivation of heterochromatin regions in differentiated cells will result in an accumulation of these long transposable transcripts, which thereby trigger an interferon-response via Toll-Like Receptors 8/9 [39]. Interestingly, ESCs and germ cells are still innocent in their TLRinduced interferon response, but once they are differentiated, the cells are no longer tolerant to long dsRNA stimulation. If the cells fail to inactivate these heterochromatin regions, this control mechanism clears these cells to prevent malignant growths (Figure 5).

Dgcr8 null ESCs have a lower proliferation rate and an absence of cardiomyogenic differentiation. Although a defect in miRNA processing might be a direct mechanism, our results suggest that Dgcr8

A
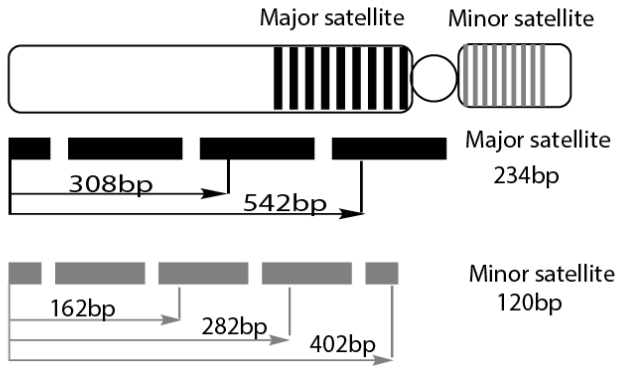

Minor satellite $120 \mathrm{bp}$

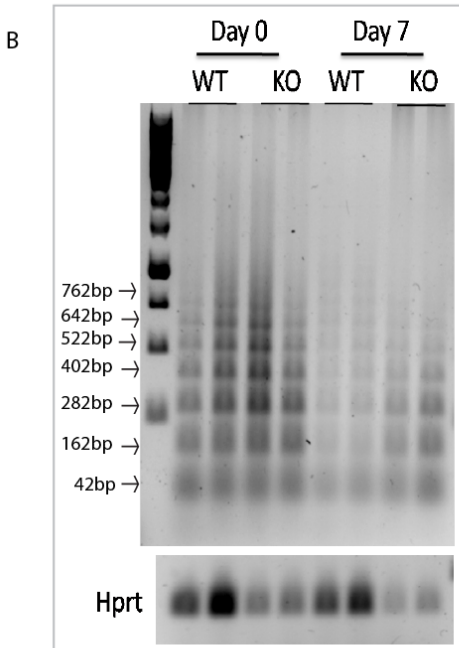

Minor satellite RNA

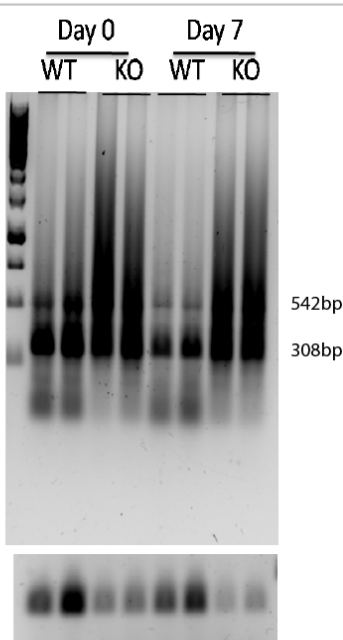

Major satellite RNA
Figure 4: Semi-quantitative RT-PCR analysis from centromeric minor satellite RNA and major satellite RNA species.

A. Illustration of the Major and Minor satellite repeat, including the different lengths of the fragment of PCR amplicons

B. RT-PCR products using Minor and Major satellite transcription specific primers to detect Minor and Major satellite RNA transcripts. PCR products are separated on DNA agarose gels with Hprt as input control.
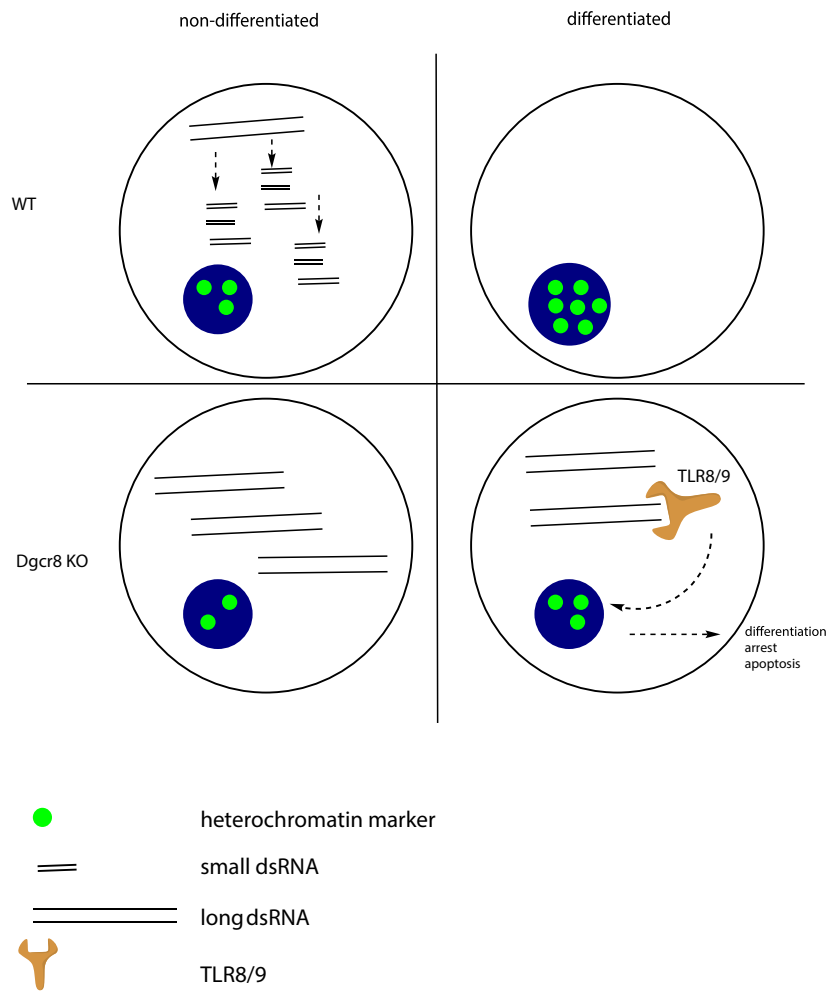

Figure 5: A working model for Dgcr8 in ESC heterochromatin silencing. Before differentiation, both WT and KO ESCs can maintain proper genome structure with moderate heterochromatin. However, KO ESCs are lacking a large population of small RNAs. Upon differentiation, in WT ESCs the endo-siRNA is blocked while $\mathrm{KO}$ cells are defective in this heterochromatin inactivation, which results in accumulation of long dsRNA. Long dsRNAs activate the interferon response via TLR8/9, leading to an arrest in differentiation and apoptosis.

is also involved in heterochromatin silencing, thereby adding another dimension to gene regulation at the epigenetic level. Furthermore, this dual role of Dgcr8 suggests that previous reported defects in Dgcr8 conditional KO mice could, in addition to a loss of small RNA biogenesis, may also be related to heterochromatin maintenance.

\section{Acknowledgements}

We thank our colleagues from Regenerative Medicine group (University Medical Center Utrecht) for their comments and suggestions on this study and Dr. Yanjuan Xu (University Medical Center Utrecht) for preparation of this manuscript.

\section{Source of Funding}

This research forms part of the Project P1.05 LUST of the research program of the BioMedical Materials institute, co-funded by the Dutch Ministry of Economic Affairs. We furthermore gratefully acknowledge the financial support of the Netherlands CardioVascular Research Initiative (CVON): the Dutch Heart Foundation, Dutch Federation of University Medical Centers, the Netherlands Organization for Health Research and Development, and the Royal Netherlands Academy of Sciences.

\section{References}

1. Sluijter JP, van Mil A, van Vliet P, Metz CH, Liu J, et al. (2010) MicroRNA-1 and -499 regulate differentiation and proliferation in human-derived cardiomyocyte progenitor cells. Arterioscler Thromb Vasc Biol 30: 859-868. [PubMed]

2. van Mil A, Vrijsen KR, Goumans MJ, Metz CH, Doevendans PA, et al. (2013) MicroRNA-1 enhances the angiogenic differentiation of human cardiomyocyte progenitor cells. J Mol Med (Berl) 91: 1001-1012. [PubMed]

3. Cai B, Li J, Wang J, Luo X, Ai J, et al. (2012) MicroRNA-124 regulates cardiomyocyte differentiation of bone marrow-derived mesenchymal stem cells via targeting STAT3 signaling. Stem cells 30: 1746-1755. [PubMed] 
Citation: Lei Z, van Mil A, van de Vrugt AM, Doevendans PA, Sluijter JPG (2015) Dgcr8 is Indispensable for Cardiac Lineage Specification in Embryonic Stem Cells. J Stem Cell Res Ther 5: 260. doi:10.4172/2157-7633.1000260

4. Glass C, Singla DK (2011) MicroRNA-1 transfected embryonic stem cells enhance cardiac myocyte differentiation and inhibit apoptosis by modulating the PTEN/Akt pathway in the infarcted heart. Am J Physiol Heart Circ Physiol 301: H2038-2049. [PubMed]

5. Chen JF, Mandel EM, Thomson JM, Wu Q, Callis TE, et al. (2006) The role of microRNA-1 and microRNA-133 in skeletal muscle proliferation and differentiation. Nat Genet 38: 228-233. [PubMed]

6. Takaya T, Ono K, Kawamura T, Takanabe R, Kaichi S, et al. (2009) MicroRNA-1 and MicroRNA-133 in spontaneous myocardial differentiation of mouse embryonic stem cells. Circ J 73: 1492-1497. [PubMed]

7. Callis TE, Pandya K, Seok HY, Tang RH, Tatsuguchi M, et al. (2009) MicroRNA$208 \mathrm{a}$ is a regulator of cardiac hypertrophy and conduction in mice. $\mathrm{J}$ Clin Invest 119: 2772-2786. [PubMed]

8. Care A, Catalucci D, Felicetti F, Bonci D, Addario A, et al. (2007) MicroRNA-133 controls cardiac hypertrophy. Nat Med 13: 613-618. [PubMed]

9. Fiedler J, Jazbutyte V, Kirchmaier BC, Gupta SK, Lorenzen J, et al. (2011) MicroRNA-24 regulates vascularity after myocardial infarction. Circulation 124 720-730. [PubMed]

10. Gurha P, Abreu-Goodger C, Wang T, Ramirez MO, Drumond AL, et al. (2012) Targeted deletion of microRNA-22 promotes stress-induced cardiac dilation and contractile dysfunction. Circulation 125: 2751-2761. [PubMed]

11. Ganesan J, Ramanujam D, Sassi Y, Ahles A, Jentzsch C, et al. (2013) MiR378 controls cardiac hypertrophy by combined repression of mitogen-activated protein kinase pathway factors. Circulation 127: 2097-2106. [PubMed]

12. van Rooij E, Sutherland LB, Liu N, Williams AH, McAnally J, et al. (2006) A signature pattern of stress-responsive microRNAs that can evoke cardiac hypertrophy and heart failure. Proc Natl Acad Sci U S A 103: 18255-18260. [PubMed]

13. Matkovich SJ, Van Booven DJ, Eschenbacher WH, Dorn GW (2011) RISC RNA sequencing for context-specific identification of in vivo microRNA targets. Circ Res 108: 18-26. [PubMed]

14. Karginov FV, Conaco C, Xuan Z, Schmidt BH, Parker JS, et al. (2007) A biochemical approach to identifying microRNA targets. Proc Natl Acad Sci U S A 104: 19291-19296. [PubMed]

15. Cesana M, Cacchiarelli D, Legnini I, Santini T, Sthandier O, et al. (2011) A long noncoding RNA controls muscle differentiation by functioning as a competing endogenous RNA. Cell 147: 358-369. [PubMed]

16. Tay Y, Rinn J, Pandolfi PP (2014) The multilayered complexity of ceRNA crosstalk and competition. Nature 505: 344-352. [PubMed]

17. Tomari Y, Zamore PD (2005) MicroRNA biogenesis: drosha can't cut it without a partner. Curr Biol 15: R61-64. [PubMed]

18. Yi R, Qin Y, Macara IG, Cullen BR (2003) Exportin-5 mediates the nuclear export of pre-microRNAs and short hairpin RNAs. Genes Dev 17: 3011-3016. [PubMed]

19. Bohnsack MT, Czaplinski K, Gorlich D (2004) Exportin 5 is a RanGTPdependent dsRNA-binding protein that mediates nuclear export of pre-miRNAs. RNA 10: 185-191. [PubMed]

20. Jiang F, Ye X, Liu X, Fincher L, McKearin D, et al. (2005) Dicer-1 and R3D1-L catalyze microRNA maturation in Drosophila. Genes Dev 19: 1674-1679. [PubMed]

21. Chendrimada TP, Gregory RI, Kumaraswamy E, Norman J, Cooch N, et al (2005) TRBP recruits the Dicer complex to Ago2 for microRNA processing and gene silencing. Nature 436: 740-744. [PubMed]

22. Chen Z, Wu J, Yang C, Fan P, Balazs L, et al. (2012) DiGeorge syndrome critical region 8 (DGCR8) protein-mediated microRNA biogenesis is essential for vascular smooth muscle cell development in mice. J Biol Chem 287: 1901819028. [PubMed]

23. Wang Y, Medvid R, Melton C, Jaenisch R, Blelloch R (2007) DGCR8 is essential for microRNA biogenesis and silencing of embryonic stem cell self-renewal. Nat Genet 39: 380-385. [PubMed]

24. Yi R, Pasolli HA, Landthaler M, Hafner M, Ojo T, et al. (2009) DGCR8-dependent microRNA biogenesis is essential for skin development. Proc Natl Acad Sci U S A 106: 498-502. [PubMed]

25. Rao PK, Toyama Y, Chiang HR, Gupta S, Bauer M, et al. (2009) Loss of cardiac microRNA-mediated regulation leads to dilated cardiomyopathy and heart failure. Circ Res 105: 585-594. [PubMed]

26. Buikema JW, Mady AS, Mittal NV, Atmanli A, Caron L, et al. (2013) Wnt/betacatenin signaling directs the regional expansion of first and second heart fieldderived ventricular cardiomyocytes. Development 140: 4165-4176. [PubMed]

27. Kanellopoulou C, Muljo SA, Kung AL, Ganesan S, Drapkin R, et al. (2005) Dicer-deficient mouse embryonic stem cells are defective in differentiation and centromeric silencing. Genes Dev 19: 489-501. [PubMed]

28. Murchison EP, Partridge JF, Tam OH, Cheloufi S, Hannon GJ (2005) Characterization of Dicer-deficient murine embryonic stem cells. Proc Nat Acad Sci U S A 102: 12135-12140. [PubMed]

29. Buhler M, Moazed D (2007) Transcription and RNAi in heterochromatic gene silencing. Nat Struct Mol Biol 14: 1041-1048. [PubMed]

30. Macias S, Plass M, Stajuda A, Michlewski G, Eyras E, et al. (2012) DGCR8 HITS-CLIP reveals novel functions for the Microprocessor. Nat Struct Mol Biol 19: 760-766. [PubMed]

31. Heras SR, Macias S, Plass M, Fernandez N, Cano D, et al. (2013) The Microprocessor controls the activity of mammalian retrotransposons. Nat Struct Mol Biol 20: 1173-1181. [PubMed]

32. Babiarz JE, Ruby JG, Wang Y, Bartel DP, Blelloch R (2008) Mouse ES cells express endogenous shRNAs, siRNAs, and other Microprocessor-independent, Dicer-dependent small RNAs. Genes Dev 22: 2773-2785.

33. Fodor BD, Kubicek S, Yonezawa M, O'Sullivan RJ, Sengupta R, et al. (2006) $\mathrm{Jmjd} 2 \mathrm{~b}$ antagonizes $\mathrm{H} 3 \mathrm{~K} 9$ trimethylation at pericentric heterochromatin in mammalian cells. Genes Dev 20: 1557-1562. [PubMed]

34. Wang Y, Baskerville S, Shenoy A, Babiarz JE, Baehner L, et al. (2008) Embryonic stem cell-specific microRNAs regulate the G1-S transition and promote rapid proliferation. Nat Genet 40: 1478-1483. [PubMed]

35. Riedmann LT, Schwentner R (2010) miRNA, siRNA, piRNA and argonautes: news in small matters. RNA Biol 7: 133-139. [PubMed]

36. Chuma S, Nakano T (2013) piRNA and spermatogenesis in mice. Philos Trans R Soc Lond B Biol Sci 368: 20110338. [PubMed]

37. Casanova M, Pasternak M, El Marjou F, Le Baccon P, Probst AV, et al. (2013) Heterochromatin reorganization during early mouse development requires a single-stranded noncoding transcript. Cell Rep 4: 1156-1167. [PubMed]

38. Malki S, van der Heijden GW, O'Donnell KA, Martin SL, Bortvin A (2014) A role for retrotransposon LINE-1 in fetal oocyte attrition in mice. Dev Cell 29: 521533. [PubMed]

39. Andersen CL, Jensen JL, Orntoft TF (2004) Normalization of real-time quantitative reverse transcription-PCR data: a model-based variance estimation approach to identify genes suited for normalization, applied to bladder and colon cancer data sets. Cancer Res 64: 5245-5250. [PubMed] 